\title{
Intra-hepatic Abscopal Effect Following Radioembolization of Hepatic Metastases
}

\author{
Maciej Powerski $^{1} \cdot$ Ralph Drewes $^{1}$ (D) Jazan Omari $^{1} \cdot$ Borna Relja $^{1} \cdot$ \\ Alexey Surov' ${ }^{1}$ Maciej Pech ${ }^{1,2}$
}

Received: 26 March 2020/Accepted: 2 August 2020/Published online: 17 August 2020

(C) The Author(s) 2020

\begin{abstract}
Purpose To search for abscopal effects (AE) distant to the site of radiation after sequential Yittrium-90 (Y-90) radioembolization (RE) of liver malignancies.

Methods and Materials In this retrospective analysis, all patients treated by RE between 2007 and $2018(n=907)$ were screened for the following setting/conditions: sequential RE of left and right liver lobe in two sessions, liver-specific MRI (MRI1) acquired max. 10 days before or after first RE (RE1), liver-specific MRI (MRI2) acquired with a minimum time interval of 20 days after MRI1, but before second RE (RE2). No systemic tumor therapies between MRI1 and MRI2. No patients with liver cirrhosis. Metastases $>5 \mathrm{~mm}$ in untreated liver lobes were
\end{abstract}

Maciej Powerski and Ralph Drewes contributed equally to this work.

Ralph Drewes

ralph.drewes@med.ovgu.de

Maciej Powerski

maciej.powerski@med.ovgu.de

Jazan Omari

jazan.omari@med.ovgu.de

Borna Relja

borna.relja@med.ovgu.de

Alexey Surov

alexey.surov@med.ovgu.de

Maciej Pech

maciej.pech@med.ovgu.de

1 Department of Radiology and Nuclear Medicine, Otto-VonGuericke University, Leipziger Str. 44, 39120 Magdeburg, Germany

2 2nd Department of Radiology, Medical University of Gdansk, Mariana Smoluchowskiego 17, 80-214, Gdansk, Poland compared in MRI1 and MRI2 and rated as follows: same size or larger in MRI2 = no abscopal effect (NAE); $>30 \%$ shrinkage without Y-90 contamination in SPECT/ $\mathrm{CT}=$ abscopal effect (AE).

Results Ninety six of 907 patients met aforementioned criteria. Median time-frame between RE1 and MRI2 was 34 (20-64) days. These 96 cases had 765 metastases which were evaluable (median 5(1-40) metastases per patient). Four patients could be identified with at least one shrinking metastasis of the untreated site: one patient with breast cancer ( 3 metastases: 0 NAE; $3 \mathrm{AE}$ ), one patient with prostate cancer (6 metastases: 3 NAE; 3 metastases $>30 \%$ shrinkage but possible Y-90 contamination) and two patients with shrinkage of one metastasis each but less than $30 \%$.

Conclusion Our retrospective study documents AE after RE of liver tumors in 1 out of 96 cases, 3 other cases remain unclear.

Keywords Abscopal effect · Radioembolization · SIRT

\section{Introduction}

Radiotherapy (RT) represents one cornerstone of the established oncological treatment regimens. Over $60 \%$ of all cancer patients receive some form of RT during their cancer treatment [1]. The general dogmatic consensus established that the efficacy of RT is exclusively limited to the induction of cancer cell death and the eradication of clonogenic survival. However, several case reports, sporadically published over the last decades, documented the 
phenomenon of tumor regression at distant sites following RT, suggesting that radiation therapy has a far-reaching or delayed influence on non-irradiated cells, i.e., the abscopal effect (AE). Immune mechanisms are the major contributor to the therapeutic outcome and any $\mathrm{AE}$ following radiation. The mode of tumor cell death through radiation is a crucial factor for immune activation-diverse phenotypes of apoptosis, necrosis, mitotic catastrophe and senescence can be observed and are reviewed elsewhere [2]. Immunogenic forms of cell deaths can convert the tumor or metastasis into an in situ vaccine by releasing damage-associated molecular patterns (DAMPs) [3-5] unleashing a cascade of recruitment, differentiation and activation of antigen-presenting cells (APC), which are responsible for priming of an anti-tumor immunity $[6,7]$. The irradiation dose, fractionation regimen and the genetic thumbprint of the irradiated cells are assumed to determine the mode of (immunogenic) cell death [2]. The clinical changes, which arise distant from the irradiated site are deemed to be the result of various factors released by the cancer cells as well as the corresponding immune cells [8]. The generation of any out-of-field-response/AE depends on whether immunosuppression or immune activation prevails in the tumor microenvironment. Several immunomodulatory substances are available to overcome immune inhibition, especially immune check-point-inhibitors have shown remarkable efficacy boosting the abscopal effects in both clinical and preclinical situations [9-11].

To our knowledge only a single report of cases, however, cover the topic of radioembolization and $\mathrm{AE}$ with or without immunotherapy [12].

Therefore, the goal of this retrospective study was to examine the occurrence and incidence of abscopal effects after the treatment of immunotherapy-naïve patients with radioembolization due to the rising clinical importance and potential exploitation of these out-of-field-effects for the improvement of tumor radiotherapy.

\section{Materials and Methods}

\section{Ethics Approval}

The study was approved by the local ethics committee.

\section{Patients}

Nine hundred and seven patients received sequential radioembolization at our department between 2007-2018 and were screened for inclusion in this retrospective study. Inclusion criteria were: (1) an existing liver MRI with adequate quality before first (MRI1) and second (MRI2) radioembolization procedure, (2) MRI1 acquisition time max. 10 days before the first radioembolization and MRI2 acquisition time min. 20 days after MRI1/first radioembolization, (3) measurable metastases in untreated liver lobe (adequate quality and sequences of liver MRI $1 \& 2$ ). Exclusion criteria were: (1) patients with hepatocellular carcinoma and liver cirrhosis, (2) immunocompromised or immunosuppressed condition, (3) chemotherapy pause less than 3 weeks before first radioembolization.

Ninety-six patients met the inclusion criteria, consented to the use of their data, and were finally included in this retrospective analysis.

\section{Evaluation Angiography and Radioembolization}

The detailed technique is described elsewhere [13]. The evaluation, i.e., mapping angiography and estimation of lung shunting, and radioembolization (RE) were performed according to our institute's standard protocols via a transfemoral access on a flat-panel detector angiography system (Artis Zeego, Siemens Healthcare, Erlangen, Germany). At our institution, RE is performed in two sequential sessions (right/left) with a time interval of 4-6 weeks between treatments. We inject SIR-Spheres Microspheres (Sirtex Medical Limited, Sydney, Australia) two weeks after evaluation at a dose adjusted to the patient's liver volume.

\section{Evaluated Parameters}

The retrospective analysis for this study was performed by two interventional radiologists with $>5$ years of experience in RE treatment in consensus using the digital subtraction angiography (DSA) and the MRI1 and MRI2 images. The Technetium macro aggregated albumin (Tc99 m-MAA) and ${ }^{90} \mathrm{Y}$ Bremsstrahlung-SPECT/CT RE treatment datasets were analyzed by a nuclear medicine specialist with several years of experience ( $>5$ years) in hybrid imaging.

\section{Statistical Analysis}

All data were retrospectively analyzed using SPSS (version 24.00, IBM Corporation, NY, USA). Descriptive statistical data are presented as whole numbers (n) and percentages of the study population. 


\section{Patient Imaging and Evaluation Parameters}

Every patient received at least two Gadolinium-ethoxybenzyl-diethylenetriamine pentaacetic acid (Gd-EOBDTPA, primovist, Bayer, Leverkusen, Germany) enhanced liver MRIs-MRI1 max. 10 days before radioembolization and MRI2 min. 20 days after MRI1/first radioembolization.

Metastases $>5 \mathrm{~mm}$ in untreated liver lobes were compared in MRI1 and MRI2 and rated as follows: same size or larger in $\mathrm{MRI} 2=$ no abscopal effect $(\mathrm{NAE}) ;>30 \%$ shrinkage and no ${ }^{90} \mathrm{Y}$ accumulation in BremsstrahlungSPECT/CT = abscopal effect (AE). Metastasis shrinkage $>30 \%$ was deemed to be $\mathrm{AE}$ and a partial response according to RECIST 1.1. Extrahepatic metastases were not evaluated for AE.

\section{Results}

After the screening of 907 RE patients 96 met the inclusion criteria (Fig. 1 Consort-diagram, Table 1). The screening parameters for $\mathrm{AE}$ after $\mathrm{RE}$ and the results are summarized in Table 2.

One certain $\mathrm{AE}$ was registered in patient A (Fig. 2, Table 2). Another patient (patient B) had a questionable AE (Fig. 3, Table 2). The reason why this AE remains questionable is potential partial contamination, based on the appearance of the Bremsstrahlung imaging, of the left liver lobe with some activity during the RE of the right liver lobe.

Furthermore, the analysis revealed two patients whose untreated metastases demonstrated noticeable shrinkage but $<20 \%$ in diameter (Fig. 4). One of these two patients had a nasopharyngeal carcinoma (Fig. 4, A-C), the other had breast cancer (Fig. 4, D-F).

\section{Discussion}

Despite the growing understanding of $\mathrm{AE}$ following radiotherapy and its underlying immune-mediated-mechanisms, its clinical incidence remains low and its triggers undeciphered. AE exist at all ages, across a variety of tumor entities and with substantial differences in radiotherapy regimens and techniques. According to a recent review, 46 case reports exist concerning $\mathrm{AE}$ from radiotherapy (1969-2014) [14]. Most reported cases of AE occur in immunogenic tumors like melanoma with high mutation burden. Immunogenicity of tumors can be modulated by high linear energy transfer (LET), which applies a high-density ionization, leading to a release of

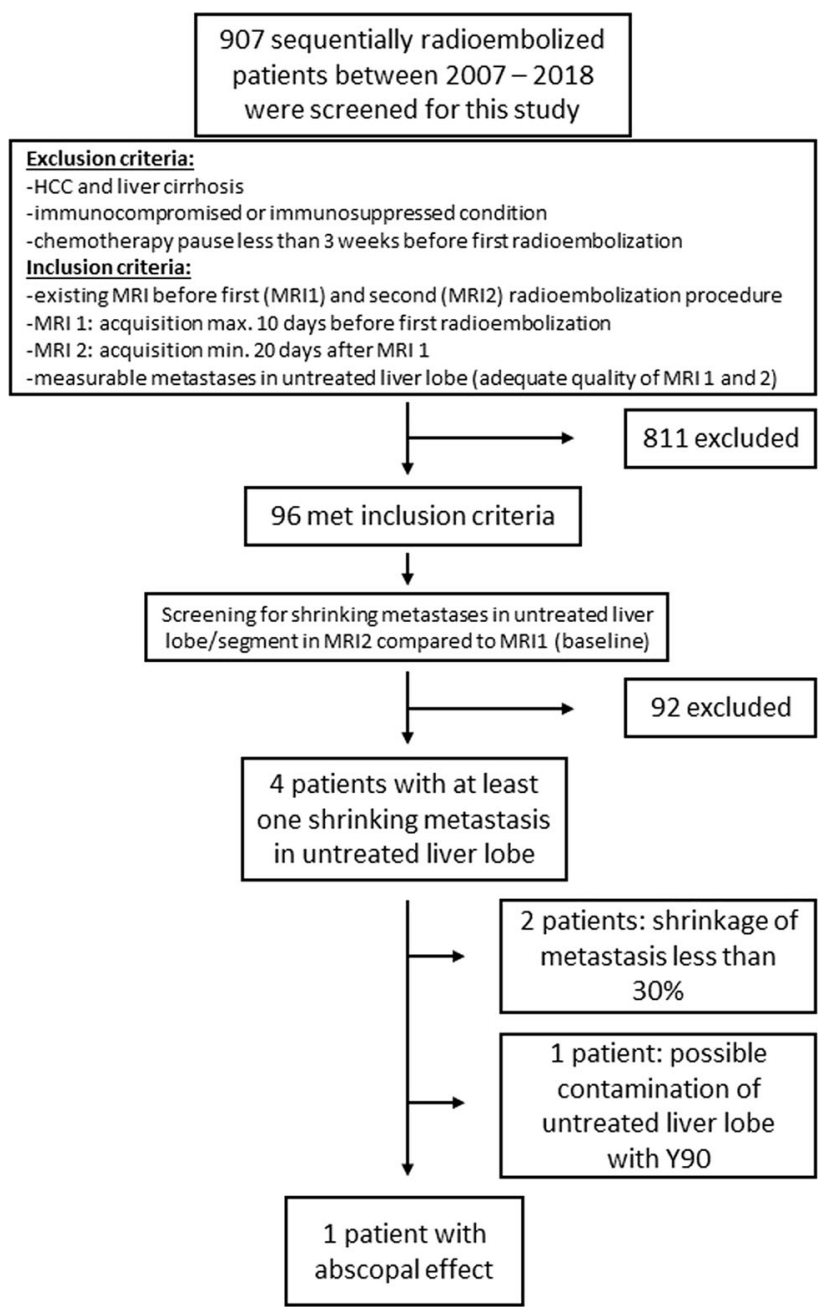

Fig. 1 Consort diagram of inclusion and exclusion criteria showing the final study population. HCC: hepatocellular carcinoma

neoantigens due to its lesser dependency on tissue oxygenation and direct DNA damage demonstrating high biological impact [15]. The heterogenous BC includes several different histological and four molecular subtypes, which have significantly different immunogenic properties [16]. Correspondingly, $\gamma$-irradiation induces different forms of cell death in $\mathrm{BC}$ depending on the genetic thumbprint and the radiation regimen [17]. In triple-negative BC, the highest concentration of DAMPs was found after high-single-dose-irradiation at 20 Gy resulting in a late onset primary necrosis with features of mitotic catastrophe and plasma membrane disintegration-especially four days after irradiation [17].

In our study, four patients demonstrated tumor-regression distant to the site treated with RE. One patient with breast cancer (patient A) showed the most noticeable tumor shrinkage. Breast cancer (BC) has generally been considered a poorly immunogenic tumor type $[16,18]$. The molecular subtype has inherent clinical relevance in terms 
Table 1 Patient characteristics

Patients screened (male/female) (n)
Age (a)*
Tumor entity $(n)$

MRI 1 - first RE (days)*

Site of first RE

Applied activity at first RE (MBq)*

MRI 1-MRI 2 (days)*

Untreated Lobe evaluated for AE in MRI 2
$96(56 / 40)$

$63.5(34-84)$

CRC 55

$\mathrm{CCC} \quad 11$

BCA

Pancreas CA

9

5

Other $(n<5)$

$1(0-9)$

Right lobe w/o Segment 4a/4b $\quad 70$

Left lobe w/o Segment 4a/4b 23

Segments

1027 (340-1900)

34 (20-64)

Right lobe w/o Segment 4a/4b 23

Left lobe w/o Segment $4 a / 4 b \quad 71$

Segments

765

$A E$ abscopal effect, $R E$ radioembolization, * median (range), $C R C$ colorectal cancer, $C C C$ cholangio cellular carcinoma, $B C A$ breast cancer; other: prostate cancer, neuroendocrine carcinoma, lung cancer, anal cancer, cervix cancer, plasmocytoma, endometrial cancer, carcinoid tumor, pharyngeal cancer, stomach cancer, duodenal cancer

of therapy (e.g., hormone receptor inhibitors) and both quantity and composition of tumor infiltrating leukocytes (12). The resulting tumor microenvironments probably affect any $\mathrm{AE}$ as well, to which extent, however, remains speculative at this point. The current evidence mostly suggests a significant correlation between immune cells and clinical outcome for estrogen receptor negative breast cancer, which does not apply to our patient $(\mathrm{PR}+, \mathrm{ER}+$, HER2-, Ki67 unknown ) (12).

The other patient (patient B) demonstrating an AE, which might however be attributed to possible Y-90 contamination during RE, had prostate cancer, a tumor entity known for its highly immunosuppressive microenvironment and low mutation burden [19].

Optimal radiation doses and fractionation regimes to induce AE with radiotherapy are still discussed controversially, and a consensus was not found up to this point, especially in combination with various immunotherapeutic options [20]. Very few researchers compare different irradiation regimes in their studies about AE. Published results are conflicting, prone to misunderstandings and difficult to compare with inconsistent usage of terminology. However, most published studies concerning this topic lean towards high-dose, hypofractionated regimes. Animal models indicate a fivefold higher $\mathrm{AE}$ incidence with high-dose, hypofractionated radiation regimes compared to the traditional (hyper-)fractionated approach [8, 21]. Radioembolization with 90-Y microspheres employs high estimated tumor doses with an average of 200-300 Gy up to a maximum of 3000 Gy [22], making it a potential initiator of any $\mathrm{AE}$ in theory, ideally catalyzed by immunotherapeutic agents. The best RT regimes to induce an AE are unknown. Growing consensus indicates that an optimal radiation dose-range likely exists, below which immune stimulation might be inefficient and above which immunosuppression predominates [23]. The ideal RE dose to initiate a sufficient or even optimal immune stimulation could be difficult to calculate, because the applied activity corresponds to liver and tumor volumes. Experience concerning $\mathrm{RE}$ and $\mathrm{AE}$ remains scarce. To our knowledge, there are only three case reports-two of which combine radio immunotherapy [12, 24, 25].

Tumor volume or metastasis mass usually leads to a different applied radiotherapeutic regime when comparing the clinical routine and the case reports of radiation-induced AE. On the one hand, lesions might be pre-treated in the clinical context-diminishing any out-of-field response; on the other hand, it was demonstrated that systemic anti-tumor immunity declines if the tumor size exceeds a certain threshold. Evolving tumors generally establish a highly immunosuppressive microenvironment infiltrated by regulatory-T-cells (Tregs), myeloid-derived suppressor cells and alternatively activated macrophages [26]. The strength of inducible anti-tumor immunity most likely depends on the tumor volume.

Radioembolization presents a rather second-tier antineoplastic treatment option usually applied as a salvage therapy in a palliative setting. The increasing interest in AE 
Table 2 Screening for abscopal effects after radioembolization

Comparison of untreated metastases in MRI 1 (baseline) and MRI 2 (follow-up after first RE)

RECIST 1.0 (96 patients)

Complete response (CR)

Partial response (PR)

Stable disease $(\mathrm{SD})$

Progressive disease (PD)

Patients with PR

Patient A (compare Fig. 2) (female, age 43, BCA)

\author{
Response of lesions (765 checked for AE) \\ Complete response -0 metastases \\ Shrinkage $>30 \%-6$ metastases ( 2 patients) \\ Shrinkage $<30 \%-2$ metastases ( 2 patients) \\ No shrinkage or progression -757 metastases
}

04/2010 - initial diagnosis of bilateral breast cancer

12/2013 - first detection of liver metastases (ER +, PR +, Her2neu-)

Endocrine therapy with Tamoxifen und GnRH analogues

Decision for radioembolization due to progressive hepatic disease

10/2015 radioembolization of the right liver lobe $+\mathrm{S} 4 \mathrm{~b}(1270 \mathrm{MBq})$

Abscopal effect registered (acquisition of MRI2 42 days after MRI1 and 43 days after first RE)

$11 / 2015$ radioembolization of the left liver lobe $(640 \mathrm{MBq})$

$5 / 16$ stable disease

Lost in further follow-up

Shrinkage of metatsases $(\mathrm{mm})$

MRI1

MRI2

$\begin{array}{lllll}18 & 19 & 31 & 14 & 14\end{array}$

19

Patient B (compare Fig. 3) (male, age 65, Prostate CA) 03/1995-initial diagnosis of prostate cancer

01/2002 - first detection of liver metastases

chemotherapy cycles with: estramustine, docetaxel, capecitabine, imatinib - until $04 / 2005$

06/2005-11/2006 multiple local ablations (interstitial brachytherapy) of liver metastases

$11 / 2007$ radioembolization of the right liver lobe $(850 \mathrm{MBq})$

Questionable Abscopal effect registered (acquisition of MRI2 42 days after MRI1 and 43 days after first RE)

01/2008 radioembolization of the left liver lobe (400 MBq)

09/2008 deceased

Shrinkage of metatsases (mm) MRI1 MRI2

$\begin{array}{llllllllll}10 & 20 & 24 & 28 & 34 & 10 & 14 & 24 & 21 & 27\end{array}$

could promote the wider RE application and enhance its status to a rather primary tool of the anti-neoplastic toolbox when combined with immunotherapeutic agents like antiCTLA-4, PD-1 antagonists, GM-CSF or IL-2. RE can effectively present tumor antigens and initiate T-cell extravasation; however, RE alone accomplishes little to overcome the suppressive tumor microenvironments. One retrospective study examined the safety of combination RE and checkpoint inhibitor immunotherapy; limited toxicity was observed and the importance to find an ideal combination protocol was emphasized [27]. The regulation of $\mathrm{AE}$ relies on a delicate balance between immune suppression and immune activation. AE is immune mediated: radiation induces local inflammation and augments T-cell activation ultimately leading to cancer cell elimination. The rarity of $\mathrm{AE}$ is attributed to the highly immunosuppressive environment of many tumor entities, resulting in increasing efforts to combine radiotherapy and immunomodulating drugs to override tumor immune evasion. The ideal time to administer immunotherapeutic agents in combination with radiotherapy remains unknown, hence the quite arbitrary application time at this point-either before, concurrent or after radiotherapy. The occurrence of AE was demonstrated to be increased by simultaneous/sequential immunotherapy [9]. Both single and fractionated radiation regimens were reported to boost $\mathrm{AE}$ combined with different immunotherapies [28-30]. Some of the most promising immunotherapies for AE are anti-CTLA4 (Ipilimumab), PD1/ PD-L1 (Nivolumab) and GM-CSF as indicated in several studies [9]. Ipilimumab, the most frequently used substance, has been demonstrated to enhance the incidence of $\mathrm{AE}$ to $25 \%$ in melanoma patients [10]. However, Ipilimumab leads to a broad systemic activation of T-cells and consequently powerful immune cell 
Fig. 2 Patient A, breast cancer, A-E, certain abscopal effect: A white arrow demonstrates the injection of Tc-99 m-MAA /the evaluation point of segments $5,6,7,8,1$ and partial 4 ; black arrow shows the evaluation point left and partial segment 4 . B application of ${ }^{90} \mathrm{Y}$ right $(5-8$, 1 and partial segment 4$)$.

C MRI1: T1w 20 min after administration of primovistlate enhancement phase 1 day prior to RE, black arrow shows metastasis prior to treatment of the right liver lobe.

D Bremsstrahlung-SPECT/CT examination: no ${ }^{90} \mathrm{Y}$ distribution in the left liver lobe. E MRI2: 42 days after the first REblack arrow shows untreated metastasis decreased in diameter

Fig. 3 Patient $B$, prostate cancer, A-E, questionable abscopal effect: A SPECT (without CT): retrospectively uncertain whether there is some ${ }^{90} \mathrm{Y}$ contamination on the left side (white arrow). B and C MRI1 and MRI2: Metastasis size regression (white arrow) in fat saturated T2w MRI. D and E MRI1 and MRI2: black arrow shows size regression in $\mathrm{T} 1 \mathrm{w}$ 20 min after administration of primovist
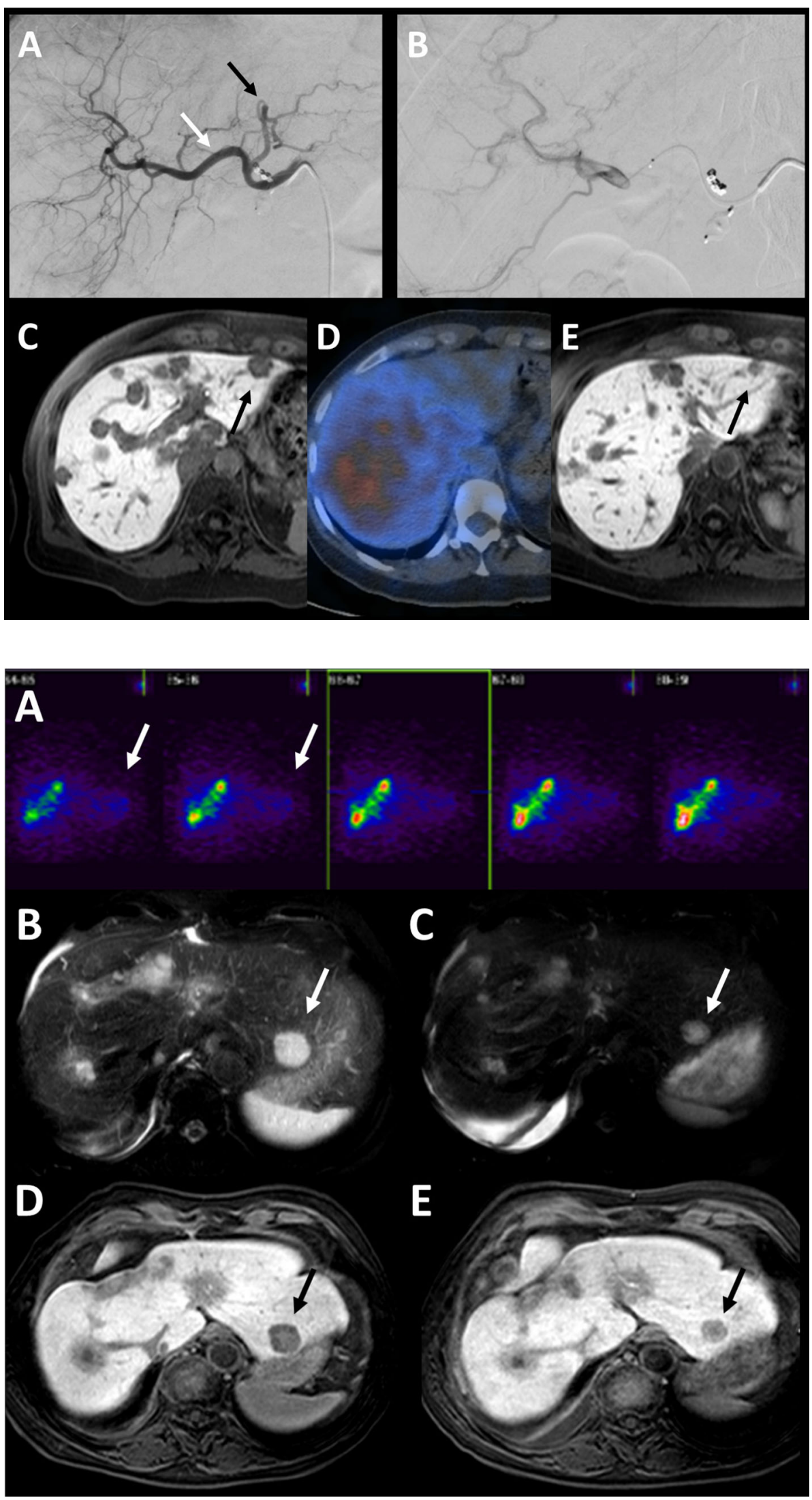
Fig. 4 Two cases of size regression $<30 \%$, but noticeably smaller, $\mathbf{A}-$

C nasopharyngeal carcinoma, D-F breast cancer: $\mathbf{A}$ and $\mathbf{C}$ ) black arrow show a metastasis decreasing from 22 to $20 \mathrm{~mm}$ (i.e., $10 \%$ ), white arrows indicate progressing lesions, e.g., 18-20 $\mathrm{mm}$ and $19-22 \mathrm{~mm}$. B Bremsstrahlung-SPECT/CT examination: RE left and segment $4 \mathrm{~b}$. Untreated segments 5-8 and 4a. D and E black arrow: $12-10 \mathrm{~mm}(\sim 20 \%)$. F Radiation damage in the liver, typical RE image for breast cancer
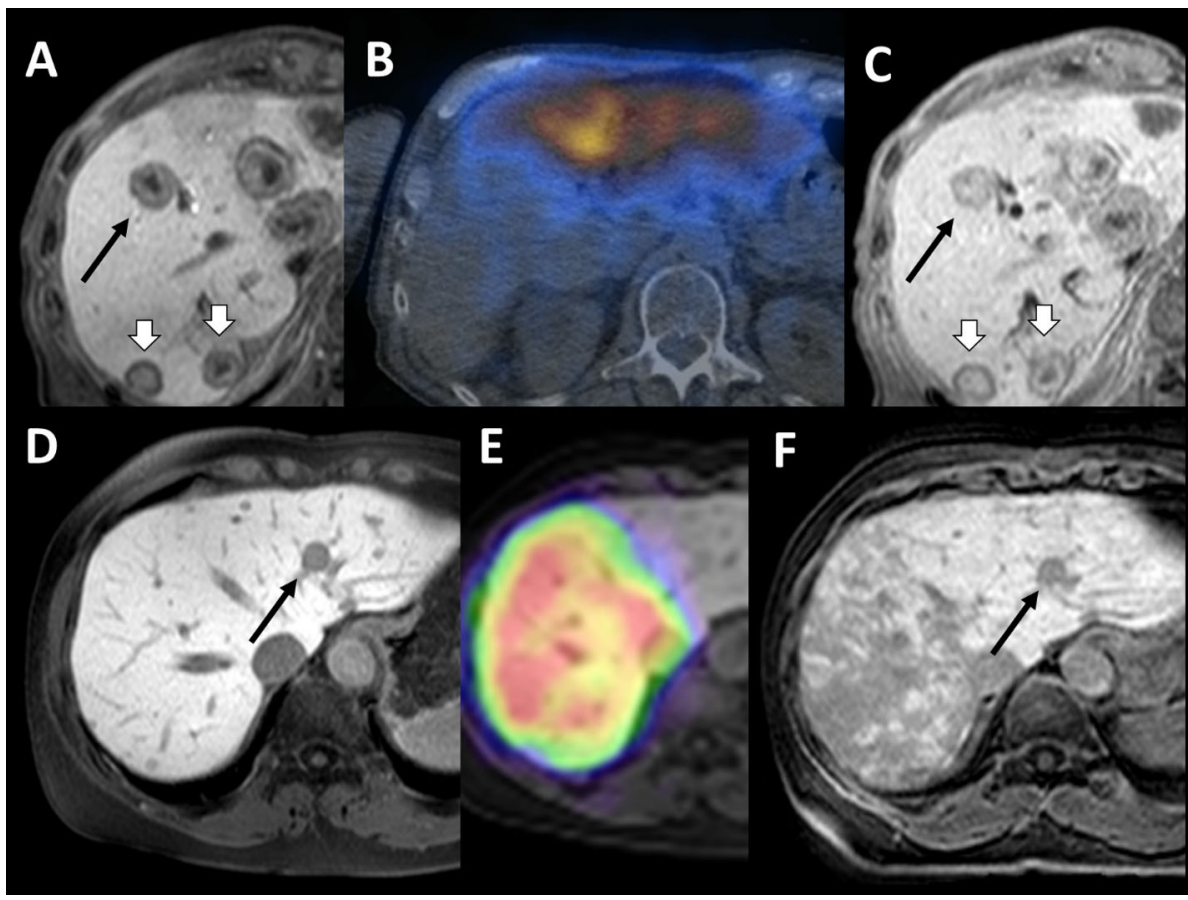

infiltration but also result severe potential side effects [31]. Another restriction is its limited tissue penetration capability [32]. T-cell activation by anti-PD-1/PD-L1 seems to be subtler. According to several studies, GM-CSF can increase the incidence of $\mathrm{AE}$ to $30 \%$ and programmed-celldeath-ligand-1 (PDL1) antibodies to 25\% [9, 11]. In our retrospective study, the observed $\mathrm{AE}$ rate without additional immunotherapy is less than $10 \%$. The targeted nature of Yttrium-90 might be suitable for the installation of immunotherapeutic agents and to initiate an antigenic cascade ultimately resulting in AE. Many ongoing studies are currently investigating the combination of radiotherapy in general and immunotherapy to boost $\mathrm{AE}$ incidences and improve treatment outcomes for various indications [33]. Preclinial trials suggest that dosage, timing and combinations are crucial when hoping for success of combined radio immunotherapy. Low immunogenicity of tumor antigens at the local site of irradiation as well as immunosuppressive cells and cytokines limit the AE even in combination approaches. Most reported cases of $\mathrm{AE}$ occur in immunogenic tumors [34]. In general, any factors that suppress the patient's immune system may prevent the development of $\mathrm{AE}$ as well as other factors like myelosuppression, overall tumor burden, lymphocyte ratio or prior exposure to radiation or cytotoxic chemotherapy [33]. In our study, patients had to discontinue their prior treatment at least four weeks before RE-still any hampering effects of prior systemic treatments are possible.

The study has several limitations. The time to observe/expect an $\mathrm{AE}$ after radiotherapy and possibly immunotherapy remains unknown. A recent review of 46 AE case reports calculated the median time of 2 months (range 0-24 months) to document an AE. In our study, we chose a relatively short timeframe with a median of 34 days (20-54) after RE to search for AE in the follow-up MRI. This was done to be certain that any potential AE effect was not caused by some other form of therapy (e.g., chemotherapy) and due to the sequential setting of RE (untreated liver lobe was scheduled for RE several weeks later). Although the ideal timeframe to expect an out-offield effect after radiotherapy remains unknown, it appears rational to assume that we missed several cases of $\mathrm{AE}$ because of the prompt follow-up in our study. Furthermore, this study evaluates only intra-hepatic and no extrahepatic AE. Systemic AE was not looked for mainly because the included patients only receive a liver MRI as an immediate short term imaging follow-up (whole-body CT was acquired much later).

Major issues, e.g., finding an optimal timeframe and radiotherapy regimen, must be solved to increase the incidence of $\mathrm{AE}$, unlock its true potential and raise its clinical importance to improve the outcome of various cancer entities. Prospective trials combining different immunotherapeutic agents and radioembolization are underway to induce a more efficient immune response.

In conclusion, although $\mathrm{AE}$ is very rare and only one certain case was identified in this study of immunotherapy naive patients, there is sufficient incentive to further examine the induction of this phenomenon. The synergy of radiotherapy/immunotherapy potentially offers the 
opportunity to generate some form of in situ cancer vaccine, which would then provide cancer "immunity" for some time.

Acknowledgements Open Access funding provided by Projekt DEAL.

Funding This study was not supported by any funding.

\section{Compliance with Ethical Standards}

Conflict of interest The authors declare that they have no conflict of interest.

Ethical Approval For this type of study formal consent is not required.

Informed Consent Informed consent was obtained from all individual participants included in the study.

Consent for Publication Consent for publication was obtained for every individual person's data included in the study.

Open Access This article is licensed under a Creative Commons Attribution 4.0 International License, which permits use, sharing, adaptation, distribution and reproduction in any medium or format, as long as you give appropriate credit to the original author(s) and the source, provide a link to the Creative Commons licence, and indicate if changes were made. The images or other third party material in this article are included in the article's Creative Commons licence, unless indicated otherwise in a credit line to the material. If material is not included in the article's Creative Commons licence and your intended use is not permitted by statutory regulation or exceeds the permitted use, you will need to obtain permission directly from the copyright holder. To view a copy of this licence, visit http://creativecommons. org/licenses/by/4.0/.

\section{References}

1. Orth M, Lauber K, Niyazi M, Friedl AA, Li M, Maihöfer C, et al. Current concepts in clinical radiation oncology. Radiat Environ Biophys. 2014;53:1-29. https://doi.org/10.1007/s00411-0130497-2.

2. Lauber K, Ernst A, Orth M, Herrmann M, Belka C. Dying cell clearance and its impact on the outcome of tumor radiotherapy. Front Oncol. 2012;2:116. https://doi.org/10.3389/fonc.2012. 00116.

3. Galluzzi L, Buqué A, Kepp O, Zitvogel L, Kroemer G. Immunogenic cell death in cancer and infectious disease. Nat Rev Immunol. 2017;17:97-111. https://doi.org/10.1038/nri.2016.107.

4. Di Blasio S, Wortel IMN, van Bladel DAG, de Vries LE, Duiveman-de Boer T, Worah K, et al. Human CD1c $(+)$ DCs are critical cellular mediators of immune responses induced by immunogenic cell death. Oncoimmunology. 2016;5:e1192739. https://doi.org/10.1080/2162402X.2016.1192739.

5. Ma Y, Adjemian S, Mattarollo SR, Yamazaki T, Aymeric L, Yang $\mathrm{H}$, et al. Anticancer chemotherapy-induced intratumoral recruitment and differentiation of antigen-presenting cells. Immunity. 2013;38:729-41. https://doi.org/10.1016/j.immuni. 2013.03.003.

6. Chen DS, Mellman I. Oncology meets immunology: the cancerimmunity cycle. Immunity. 2013;39:1-10. https://doi.org/10. 1016/j.immuni.2013.07.012.
7. Brix N, Tiefenthaller A, Anders H, Belka C, Lauber K. Abscopal, immunological effects of radiotherapy: narrowing the gap between clinical and preclinical experiences. Immunol Rev. 2017;280:249-79. https://doi.org/10.1111/imr.12573.

8. Filatenkov A, Baker J, Mueller AMS, Kenkel J, Ahn G-O, Dutt S, et al. Ablative tumor radiation can change the tumor immune cell microenvironment to induce durable complete remissions. Clin Cancer Res. 2015;21:3727-39. https://doi.org/10.1158/10780432.CCR-14-2824.

9. Golden EB, Chhabra A, Chachoua A, Adams S, Donach M, Fenton-Kerimian M, et al. Local radiotherapy and granulocytemacrophage colony-stimulating factor to generate abscopal responses in patients with metastatic solid tumours: a proof-ofprinciple trial. Lancet Oncol. 2015;16:795-803. https://doi.org/ 10.1016/S1470-2045(15)00054-6.

10. Chandra RA, Wilhite TJ, Balboni TA, Alexander BM, Spektor A, Ott PA, et al. A systematic evaluation of abscopal responses following radiotherapy in patients with metastatic melanoma treated with ipilimumab. Oncoimmunology. 2015;4:e1046028. https://doi.org/10.1080/2162402X.2015.1046028.

11. Twyman-Saint Victor C, Rech AJ, Maity A, Rengan R, Pauken KE, Stelekati E, et al. Radiation and dual checkpoint blockade activate non-redundant immune mechanisms in cancer. Nature. 2015;520:373-7. https://doi.org/10.1038/nature14292.

12. Ghodadra A, Bhatt S, Camacho JC, Kim HS. Abscopal effects and yttrium-90 radioembolization. Cardiovasc Intervent Radiol. 2016;39:1076-80. https://doi.org/10.1007/s00270-015-1259-0.

13. Salem R, Thurston KG. Radioembolization with 90Yttrium microspheres: a state-of-the-art brachytherapy treatment for primary and secondary liver malignancies. Part 1: Technical and methodologic considerations. J Vasc Interv Radiol 2006;17:1251-78. https://doi.org/10.1097/01.RVI.0000233785. 75257.9A.

14. Abuodeh Y, Venkat P, Kim S. Systematic review of case reports on the abscopal effect. Curr Probl Cancer. 2016;40:25-37. https:// doi.org/10.1016/j.currproblcancer.2015.10.001.

15. Gorin J-B, Ménager J, Gouard S, Maurel C, Guilloux Y, FaivreChauvet A, et al. Antitumor immunity induced after $\alpha$ irradiation. Neoplasia. 2014;16:319-28. https://doi.org/10.1016/j.neo.2014. 04.002.

16. Hammerl D, Smid M, Timmermans AM, Sleijfer S, Martens JWM, Debets R. Breast cancer genomics and immuno-oncological markers to guide immune therapies. Semin Cancer Biol. 2018;52:178-88. https://doi.org/10.1016/j.semcancer.2017.11. 003.

17. Hennel R, Brix N, Seidl K, Ernst A, Scheithauer H, Belka C, et al. Release of monocyte migration signals by breast cancer cell lines after ablative and fractionated $\gamma$-irradiation. Radiat Oncol. 2014;9:85. https://doi.org/10.1186/1748-717X-9-85.

18. Denkert C. The immunogenicity of breast cancer-molecular subtypes matter. Ann Oncol. 2014;25:1453-5. https://doi.org/10. 1093/annonc/mdu235.

19. De Velasco MA, Uemura H. Prostate cancer immunotherapy: where are we and where are we going? Curr Opin Urol. 2018;28:15-24. 0000000000000462 .

20. Rödel F, Frey B, Multhoff G, Gaipl U. Contribution of the immune system to bystander and non-targeted effects of ionizing radiation. Cancer Lett. 2015;356:105-13. https://doi.org/10.1016/ j.canlet.2013.09.015.

21. Camphausen K, Moses MA, Ménard C, Sproull M, Beecken W-D, Folkman J, et al. Radiation abscopal antitumor effect is mediated through p53. Cancer Res. 2003;63:1990-3.

22. Kennedy AS, Nutting C, Coldwell D, Gaiser J, Drachenberg C. Pathologic response and microdosimetry of (90)Y microspheres in man: review of four explanted whole livers. Int J Radiat Oncol 
Biol Phys. 2004;60:1552-633. https://doi.org/10.1016/j.ijrobp. 2004.09.004.

23. Bernstein MB, Krishnan S, Hodge JW, Chang JY. Immunotherapy and stereotactic ablative radiotherapy (ISABR): a curative approach? Nat Rev Clin Oncol. 2016;13:516-24. https://doi.org/ 10.1038/nrclinonc.2016.30.

24. Adashek JJ, Salgia M, Dizman N, Kessler J, Pal SK. Concomitant radioembolization and immune checkpoint inhibition in metastatic renal cell carcinoma. Case Rep Oncol. 2018;11:276-80. https://doi.org/10.1159/000489995.

25. Deipolyi AR, Bromberg JF, Erinjeri JP, Solomon SB, Brody LA, Riedl CC. Abscopal effect after radioembolization for metastatic breast cancer in the setting of immunotherapy. J Vasc Interv Radiol. 2018;29:432-3. https://doi.org/10.1016/j.jvir.2017.10. 007.

26. Motz GT, Coukos G. Deciphering and reversing tumor immune suppression. Immunity. 2013;39:61-73. https://doi.org/10.1016/j. immuni.2013.07.005.

27. Zhan C, Ruohoniemi D, Shanbhogue KP, Wei J, Welling TH, Gu $\mathrm{P}$, et al. Safety of combined Yttrium-90 radioembolization and immune checkpoint inhibitor immunotherapy for hepatocellular carcinoma. J Vasc Interv Radiol. 2020;31:25-34. https://doi.org/ 10.1016/j.jvir.2019.05.023.

28. Dewan MZ, Galloway AE, Kawashima N, Dewyngaert JK, Babb JS, Formenti SC, et al. Fractionated but not single-dose radiotherapy induces an immune-mediated abscopal effect when combined with anti-CTLA-4 antibody. Clin Cancer Res.
2009;15:5379-88. https://doi.org/10.1158/1078-0432.CCR-090265 .

29. Deng L, Liang H, Burnette B, Beckett M, Darga T, Weichselbaum RR, et al. Irradiation and anti-PD-L1 treatment synergistically promote antitumor immunity in mice. J Clin Invest. 2014;124:687-95. https://doi.org/10.1172/JCI67313.

30. Marconi R, Strolin S, Bossi G, Strigari L. A meta-analysis of the abscopal effect in preclinical models: Is the biologically effective dose a relevant physical trigger? PLoS ONE. 2017;12:e0171559. https://doi.org/10.1371/journal.pone.0171559.

31. Chen H, Liakou CI, Kamat A, Pettaway C, Ward JF, Tang DN, et al. Anti-CTLA-4 therapy results in higher CD4+ICOShi T cell frequency and IFN-gamma levels in both nonmalignant and malignant prostate tissues. Proc Natl Acad Sci USA. 2009;106:2729-34. https://doi.org/10.1073/pnas.0813175106.

32. Patel SP, Woodman SE. Profile of ipilimumab and its role in the treatment of metastatic melanoma. Drug Des Devel Ther. 2011;5:489-95. https://doi.org/10.2147/DDDT.S10945.

33. Kang J, Demaria S, Formenti S. Current clinical trials testing the combination of immunotherapy with radiotherapy. J Immunother Cancer. 2016;4:51. https://doi.org/10.1186/s40425-016-0156-7.

34. Blankenstein T, Coulie PG, Gilboa E, Jaffee EM. The determinants of tumour immunogenicity. Nat Rev Cancer. 2012;12:307-13. https://doi.org/10.1038/nrc3246.

Publisher's Note Springer Nature remains neutral with regard to jurisdictional claims in published maps and institutional affiliations. 